International Research Journal of Diabetes and Metabolism
(DOI:10.28933/IRJDM)

\title{
Effects of oral antidiabetics on cancer incidence and mortality
}

\author{
Neto, P. P. M $^{1}$; Silva, M. R. P $^{2}$; Soares, C. L. R $^{3}$; Lucena, C. C. $\mathbf{O}^{4}$; Pereira, P. S $^{5}$; Silva, T. $\mathbf{G}^{6}$
}

1Postgraduate Program of Biological Sciences, Federal University of Pernambuco - UFPE, 2 Undergraduate student of Biological Sciences, Federal University of Pernambuco - UFPE; 3Postgraduate Program of Pharmaceutical Sciences, Federal University of Pernambuco - UFPE; 4 Postgraduate Program of Northeast Biotechnology Network - RENORBIO, Federal University of Pernambuco - UFPE; 6 Professor/Researcher on Department of Antibiotics, Federal University of Pernambuco - UFPE.

Introduction: Type 2 diabetes mellitus is a chronic progressive disease that can be controlled by any oral hypoglycemic, with biguanides, thiazolidinediones and sulfonylureas being the main classes of these drugs in the pharmaceutical market. Epidemiological analyzes suggest that diabetics are at higher risk of developing tumors than normoglycemics. A large number of studies have shown the link between oral antidiabetic drugs and developmental rates and mortality in cancer cases. Objective: To present clinical and preclinical results on the relationship between oral hypoglycemic agents and cancer. Methodology: A bibliographical survey was carried out of the main academic journals, with prevalence of articles from the last five years. Results and Discussion: Metformin is a biguanide that phosphorylates hepatic cyclic AMP, a mechanism also proposed as antineoplastic. Epidemiological studies associate the use of metformin with the lower incidence of hepatocellular and pancreatic cancers in diabetic patients. Other studies have shown a $21 \%$ lower mortality rate in women with breast cancer and $24 \%$ lower in men with prostate cancer who took metformin. Thiazolidinediones are receptor agonists, predominantly present in adipocytes, capable of increasing glucose uptake and improving insulin sensitivity. Randomized trials have shown that pioglitazone was associated with a significant reduction in breast cancer, however, there are concerns regarding its use related to the increase in the incidence of bladder cancer. Sulphonylureas, on the other hand, increase insulin secretion by pancreatic $\beta$ cells. In an observational study, glibenclamide was associated with an increased risk of $20 \%$ to *Correspondence to Author:

Neto, P. P. M

Postgraduate Program of Biological Sciences, Federal University of Pernambuco - UFPE

How to cite this article:

Neto, P. P. M; Silva, M. R. P; Soares, C. L. R; Lucena, C. C. O; Pereira, P. S; Silva, T. G. Effects of oral antidiabetics on cancer incidence and mortality.International Research Journal of Diabetes and Metabolism, 2018, 1:4 $25 \%$ in the overall incidence of cancer. Conclusion: The use of oral hypoglycemic agents may decrease the incidence and mortality of certain types of cancer. However, more studies are needed to elucidate its possible effects on the neoplastic process.

Keywords: Cancer; Diabetes; Oral Hypoglycemic Agents 